\title{
The Tibetan Wisdom of Isaac Eliaz, M.D., M.S., L.Ac.
}

\section{Russ Mason, M.S.}

$I$ saac Eliaz, M.D., M.S., L.Ac. (Amitabha Medical Clinic and Healing Center, Sebastopol, California), has been a pioneer in the field of integrative medicine since the early 1980s. His guiding mission has been the integration of multiple healing modalities-from both ancient and modern paradigms - into a holistic practice of medicine. This synergistic integration is the very heart of his clinical practice and research. Dr. Eliaz developed - and was granted patents for-several herbal formulations. He is a frequent lecturer on integrative medical approaches to health, immune enhancement, and cancer prevention and treatment. Dr. Eliaz has also taught courses on Traditional Chinese Medicine for medical doctors and for licensed acupuncturists.

In order to substantiate nutritional approaches to health, Dr. Eliaz participates regularly in clinical studies and has had papers published in peer-reviewed journals. In addition, Dr. Eliaz' formulations have been submitted for validation in independent human clinical studies that have had results published in peer-reviewed journals.

Russ Mason: You have an unusual background-as an individual and as a physician. Please talk about that.

Isaac Eliaz: I am originally from Israel but grew up in many different countries during the course of my childhood. As such, I was exposed to different forms of alternative medicine. My father was an agricultural engineer for developing countries and traveled often. I would accompany him on these trips and so I lived in Africa for a year, later in Brazil, and still later, in Korea.

I was 15 years old, living in Korea, when I began to study martial arts and yoga. It was at that time I became interested in alternative healing. Later, while I was in medical school in Israel-a 7-year program-I became a yoga teacher and learned Shiatsu and Japanese manipulative therapy. While attending medical school full time I also took a 3-year course in Chinese medicine, thus pursuing two parallel courses of study in the healing arts. I also studied Western herbology for 1 year at the Hebrew University of Jerusalem.

Upon graduation from medical school, I was privileged to teach Chinese medicine to allopathic doctors as part of a project that I organized with the Hebrew University of Jerusalem. By then, I had established a healing center in Israel. I then felt that it was time for me to continue my studies abroad. I came to the United States, where I earned a M.S. degree in Chinese medicine and became a licensed acupuncturist. I have a separate license as a Chinese doctor.

The greatest influence in my life was the study of Tibetan Buddhism, and it remains an ongoing influence. My study of Tibetan Buddhism has a strong bearing on the way I treat patients, especially those with cancer.

RM: What is it about Tibetan Buddhism that makes it especially applicable to the treatment of cancer?

IE: It is the wisdom and perception of the impermanence of life and death. My view of integrative medicine with respect to cancer is to apply the path of knowledge and wisdom.

The knowledge is the information that we gather as health providers as well as patients. It is a linear conceptual process of reading and learning that allows us to expand the scope of health care and to integrate various tools and systems into a more effective health care regimen. The wisdom of integrative medicine is nonlinear and nonconceptual. It requires both the practitioner and the patient to relax and to let go of preconceived concepts.

\section{RM: Please explain a little further about that.}

IE: While the knowledge is conceptual information that we learn, the wisdom, however, is a nonconceptual process of learning; it happens only when one is ready for it. And, if one is able to integrate this into his or her practice, it can develop into a very powerful tool for both patient and practitioner. It's not what you do but how you do it.

RM: How does this apply to treating patients with cancer?

IE: When we face a significant health challenge, such as cancer, it creates a shakeup in our systems. Things that used to trouble us are pushed to the sidelines. We suddenly face life-and-death issues and the possibility of departing from this human life. Wisdom can be found when we slow down and get in touch with our cores.

The goal of integrative medicine, especially with cancer, is to nourish both this new wisdom and the strengthening of our cores from diagnosis through the remainder of our lives no matter what the "medical outcome" [may be]. When we engage in the path of knowledge and wisdom, there is no possible outcome but a successful outcome regardless of the result of the procedures or the 


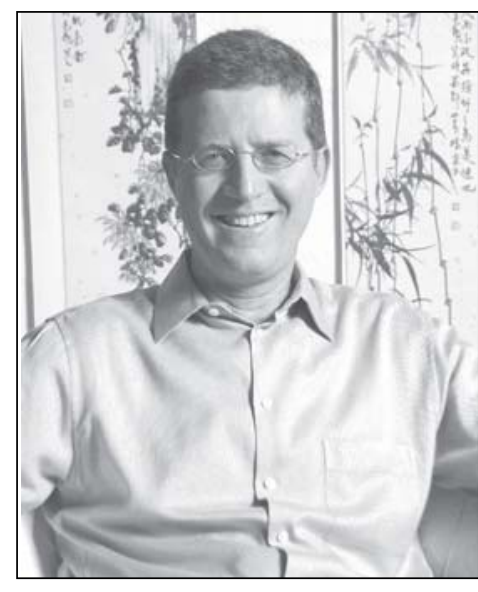

Isaac Eliaz, M.D., M.S., L.Ac., Amitabha Medical Clinic and Healing Center. Photo courtesy of Isaac Eliaz, M.D., M.S., L.Ac.

for many years and I rely on it in my practice. It is a remarkable tool for diagnosis as well as prognosis.

RM: Is pulse diagnosis something that you recommend to other health care practitioners?

IE: Yes, but there are different levels. On a technical, scanning level, it's not too difficult to learn. But to learn in a more profound way-that is, to learn about the person's past, the present, and maybe where the person is going; and to learn about the person's relationship between health and disease-that can take a lifetime of learning. I have been practicing pulse diagnosis for over 20 years, and feel that I am still at the beginning.

RM: Some years ago I interviewed a Tibetan doctor, and he said that it was possible to diagnose a liver ailment with pulse diagnosis. Do you agree?

IE: Oh yes. That is relatively simple. For example, a patient might have experienced a trauma that affected his lungs when he was 8 years old, and the injury never healed. This trauma might still be with that child as an adult and could be affecting his current health. This is the sort of thing that practitioners can obtain from the pulse.

\section{RM: You can get all that information from a pulse?}

IE: Yes. But the question is: How does one get this information? It seems that the "big picture" gets lost because we live in a world of specialization. Our minds become focused on details, on one aspect of health, or the patient. To see the big picture, we need to relax, to let go. Few people-including doctors-have time to really relax.

Let's use the visual image of a circle to explain my point. It is the image that best reflects integrative medicine in Chinese medicine. The circle reflects endless possibilities. One more thing can always be added to the circle, and all the areas of the circle are equal. As a doctor adds more therapies and protocols to his or education, you might imagine that doctor filling the circle.

A Western medical doctor who realizes that he or she cannot offer what the patient needs most may begin to study herbs, chelation, diet, and supplements. As the doctor becomes more skilled, he or she begins to "fill in the circle." But even with these newly acquired skills and the Western physician's mindset, the interaction with the doctor's patient did not really change. So, in this sense, the doctor is still limited. It's using different tools with the same allopathic thinking.

However, if that doctor were to deepen his or her meditation practice, and develop intuition, then you might imagine the circle becoming bigger and bigger. This reflects the greater depth of possibility and effectiveness of his or her treatments.

RM: Please explain your views about integrative medicine.

IE: First, integrative medicine is a patient-driven medicine. Today I am a doctor; tomorrow I may be a patient. This means that the doctor and the patient must come to an interaction on equal ground. For example, I don't give new patients questionnaires when they come to my clinic. I ask them to write down what they think is important. I give them some guidelines, of course, and these guidelines cover certain basic areas such as nutrition, medications, supplements, and so forth. I ask the patients to determine what is important to them-what they choose to divulge and how they do so provides me with valuable insight.

This process begins when they write their histories based on their priorities, and it continues throughout their experiences in the clinic. In the first interaction, we do not diagnose a disease. We merely try to identify life patterns where the disease is just an outcome.

\section{RM: Will you explain that a little more?}

IE: If an individual has a certain life pattern there will be consequences as part of the specific life pattern. The disease is simply a sign along the road, with the road being a life's journey. One consequence might be heart disease, or prostate cancer.

Currently, 80 percent of my practice is with people with cancer. In [conventional] cancer care, the patient goes to a cancer center to see an oncologist. The patient is given a gown, which is open in the back and which was washed so often it has lost its color. A patient can lose his or her entire personality wearing one of those gowns. There is no more Russ; there is no more Isaac. Then the physician enters fully dressed. Right there the patient is reduced to almost nothing.

The patient is also fearful because he or she has just been diagnosed with cancer; and is feeling the pressure of, "you must do this or that or else you will die in 6 months!" The patient has no chance to breathe. He or she is immediately drawn into the system and started on treatments. 
This is especially important in cancer treatment because, when a person is diagnosed with cancer, all of his or her priorities shift completely. What was important a few seconds before the diagnosis has now become trivial; the situation is one of life and death, and one is vulnerable. [Conventional] oncology can be about fear and having a finite time to live.

Sometimes I see patients who initially refuse chemotherapy eventually realizing that chemo is the appropriate treatment. I give them the space to make their decisions and help them navigate through the various alternatives. Sometimes patients will succumb to pressure doctors put on them and agree to treatments they really don't want to do. I consciously avoid doing this in my practice, giving a different quality to the interaction; thus the patient opens his or her heart. That is the first principle. The second principle is to be nonbiased and nonjudgmental. This doesn't mean that one is nondiscriminative; it is important to discriminate what makes sense and what doesn't. But one must suspend judgment and have an open mind-it is a very complex task.

\section{RM: How does one suspend judgment?}

IE: From a conceptual point of view, it's almost impossible. But one must first ask, what is the origin of perception? Humans have a habit of grasping at perceptions, thoughts, and sensory appearances. And we often attach an emotional response to the perception: we like this food, we dislike that food; we like this person, we have an aversion to the other person; et cetera. These are emotional biases; we either desire something or have an aversion to something. So, from this point of view, it is very difficult not to have judgment.

We can try to eliminate it from a nonconceptual approach and through meditation, where the mind becomes more relaxed, more open, beyond a pinpoint view of an object. If the mind relaxes and sees the big picture, it is not because of conceptual grasping but because one is relaxed. When the mind relaxes, the habit of grasping starts to loosen up. We want it to occur at the first consultation. It can happen when someone does acupuncture, or chiropractic, or when giving an injection or an IV. If someone is open, then anything can happen.

We know, from quantum physics, that nothing is solid. Science knows this. But, it is our perception of our reality as solid, our grasping, and our mental tightness that prevents spontaneous change. The optimum opportunity for healing occurs when both the practitioner as well as the patient are relaxed and open, allowing the process to peel off as many layers as possible without resistance, and get to the essence, the spaciousness beyond grasping and clinging.

RM: Are there direct, practical benefits for your patients because of your own meditation practice?

IE: Yes. I see my patients in a more holistic way. I become more compassionate, more patient. There is a greater mindfulness that allows me to better understand and benefit the patient. The ability to see profoundly and holistically comes from wisdom and there has to be a balance between the two: from reading and studying and from meditating and relaxing.
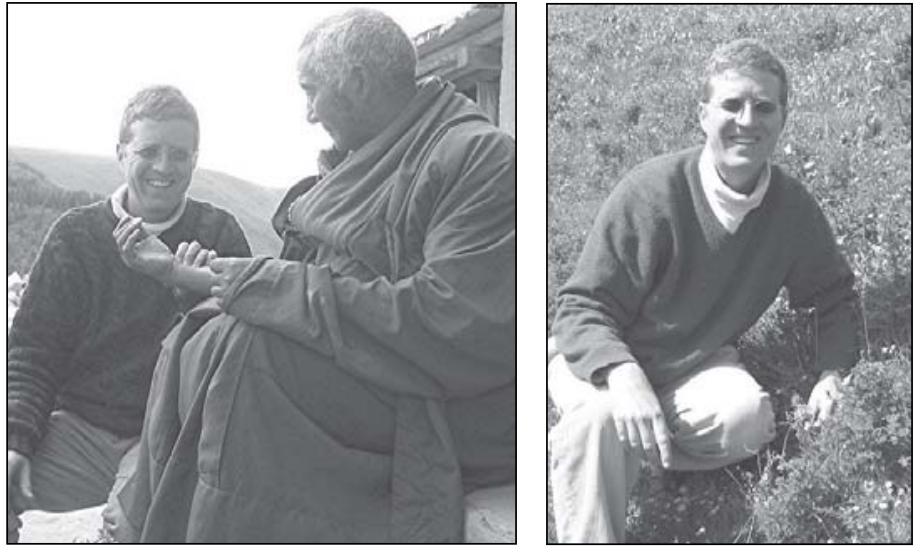

Dr. Eliaz with a Tibetan monk and relaxing outdoors. Photos courtesy of Isaac Eliaz, M.D., M.S., L.Ac.

\section{To Contact Dr. Isaac Eliaz}

Isaac Eliaz, M.D., M.S., L.Ac.

Amitabha Medical Clinic and Healing Center

7064 Corline Court, Suite A

Sebastopol, CA 95472

Phone: (707) 829-5900

Fax: (707) 829-5282

Website: www.dreliaz.com/

For information on Dr. Eliaz' dietary supplements, contact: Website: http://www.econhealth.com/

Phone (800) 308-5518

RM: What would you say to health care providers who are very busy, who have no time to meditate?

IE: I would say first, slow down. There are many different kinds of meditations to calm the mind-one simple one by focusing on one thing, such as breathing. This will slowly reduce the background noise and the mind will become quieter. At first, there will be many thoughts, with some gaps of quietness. Then the gaps will become longer, until you are able to look inside, and develop "insight."

Interestingly, for patients, calm mind (Shamata) meditation can counter side-effects of chemotherapy and it is more effective for depression than antidepressant drugs. For health care practitioners, meditation allows us to see patients better and offer more compassionate and effective care.

I'm somewhat different in my background and training. Many physicians study integrative, or alternative, medicine after they've finished their allopathic studies. My training was parallel, and I explored alternative healing systems while very young. Although I am a Western physician, I have had extensive medical training in Chinese medicine. I continue to study to this day, with a focus on balancing knowledge and wisdom. It's an endless path. While you make progress, you continue to realize that you are still a beginner.

\section{RM: What are you studying now?}

IE: Since coming to the States, I have been training in Traditional Chinese Medicine, classical homeopathy, naturopathy, 
CranioSacral therapy and Tibetan Medicine. I could drown in all of this information if I didn't have the ability to access passive wisdom, by relaxing and using nonconceptual approaches. That is an important message I always share in my lectures and in my enewsletter. [See box entitled To Contact Dr. Isaac Eliaz in order to reach him for information about these.]

RM: What are the benefits of studying all these various types of therapies?

IE: The main benefit is that you arrive at a highly individualized medicine focusing on the individual and not the disease. If herbs are more appropriate for that individual then that is something I can offer; if a prescription drug is helpful, I can offer that too.

Allopathic medicine is highly protocol-driven, especially in oncology. If one treatment has a 70 percent versus another at a 40 percent success rate, but through an individualized assessment you find out that the person will respond to the 40 percent protocol, you can give that patient the 40 percent protocol. A patient seeing a regular oncologist will never get that more appropriate treatment because it's considered to be less effective.

The idea of individualization considers gender, age, geographic location, time of year/day, ethnic background, religious background, socioeconomic background, et cetera-all of which should be taken into consideration.

This philosophy of individualizing approaches can be applied to our lives, our clinical environment, and our approach to formulating new supplements and herbal preparations. That is why some of the Eastern preparations have such remarkable efficacy with comparatively small dosages of herbs. This is something I have learned to respect.

\section{RM: Please give me an example from Tibetan medicine?}

IE: One well-known herbal preparation based on a traditional Tibetan formula called Gambar 25 is Padma Basic ${ }^{\circledR}$ [EcoNugenics, Santa Rosa California].* It's an approved medication for peripheral artery diseases in Switzerland and other countries. The health benefits are extraordinary; it improves circulation, reduces inflammation, and regulates abnormal immune response.

The reason one preparation can be effective for so many health conditions is that it addresses many underlying patterns that can affect our health. It is amazing that Tibetan medicine knew about it and created such a formulation hundreds of years ago.

It works by regulating inflammatory response, reducing hyperviscosity, and enhancing the immune system, allowing for a better clinical response. This is significant because we are now discovering that inflammation is the basis for almost every

*Disclosure: Dr. Eliaz founded this company. chronic illness-coronary disease, stroke, memory loss, cancer, peripheral artery disease, cognitive deterioration, autoimmune diseases and, of course, infections. Therefore, regulating inflammation will benefit the overall health and longevity of the person, as the Tibetans predicted.

RM: In addition to seeing patients, are you actively working in creating beneficial new supplements? If so, what are you working on now?

IE: Yes. Currently I am working on an oral detoxification preparation that will bind heavy metals and remove them from the body. I have been working on a compound called Modified Citrus Pectin (MCP) for about 10 years now.

\section{RM: Please explain about that.}

IE: Pectin is a structural cell-wall polymer in the peels of citrus fruits that is composed mainly of galacturonic acid. By modifying this large molecule to a specific structure and size, it can be made to bind to cancer cells and prevent them from metastasizing in the circulatory system. The molecule can also prevent angiogenesis, which is one of the mechanisms of tumor nourishment and growth.

\section{RM: What is the mechanism for pectin?}

$\mathbf{R M}$ : The mechanism is through a group of proteins expressed on the surface of many types of cancer cells called galectins. These cell-wall surface proteins contain an amino-acid chain inside the cell and a galactose residue on the outside. These proteins occur in low density in every cell. The number and density increases as cancer cells become dysplastic, malignant, and aggressive. This molecule facilitates communication between cancer cells. They create new colonies, new blood-vessel cells, and endothelial cells. These interactions have been well-characterized.

We have learned, using MCP in our studies and clinical trials, that it can slow down the primary tumor as well as the metastatic process. This was studied in men with recurrent prostate cancer. In the cases where the prostate was removed to an undetectable level of PSA, the PSA level started to rise again, indicating biochemical recurrence of the cancer. When we administered MCP, we saw a significant decrease in the rate of increase in PSA in most patients. MCP is still underutilized, but there is enough data to merit its use.

MCP has another important application-its ability to bind heavy metals, such as mercury, lead, cadmium, and arsenic. When pectin is properly modified, it can easily complex divalent cations and encourage excretion.

The efficacy of pectates was known, going back to the time of Chernobyl (the nuclear disaster in Byelorussia, which occurred in 1987). There was a published paper that reported cases with children who were exposed to high levels of radioactive readings and 
given pectates to "bind" some of the radioactive material. Consequently, radioactive readings among these children declined by 50-60 percent. As this was very impressive, I reasoned that, if I could modify the pectin to a low molecular weight, it would get absorbed into the bloodstream and then work as a gentle, systemic chelator that could chelate heavy metals on an ongoing basis.

\section{RM: Please explain why chela-} tion is necessary.

IE: All of us have some level of heavy metal toxicity; there is no way to escape it. We are exposed to toxic metals every day and they accumulate in our bodies. Chelation of heavy metals is important because heavy metals adversely affect every system in the body and contribute to medical conditions including cancer, cardiovascular disease, neurologic conditions (including autism), immune suppression, behavioral changes, and other conditions.

While, [conventionally] used chelators, such as DMPS [sodium 2,3-dimercaptopropane-l-sulfonate], DMSA [dimercaptosuccinic acid] (prescription drugs), or ETDA [ethylenediaminetetraacetic acid] chelate essential minerals these drugs cause undesirable side-effects. There is a strong need for a systemic chelator that can be used continuously for long-term health without side-effects.

RM: Please give me an example of heavy metal exposure and how MCP will chelate the metal away.

IE: Imagine you have tuna or seafood for dinner. Virtually all tuna contains some level of mercury as well as most fatty fish. If you have MCP in your system already, it will bind to the mercury and protect you. This is extremely important.

RM: Have there been any studies on MCP and its effectiveness as a chelator?

IE: Yes. We have conducted two clinical trials. In those trials, we monitored the urinary excretion of heavy metals over 24 hours as a baseline and, again, after taking MCP. We demonstrated that MCP was able to increase the urinary excretion of mercury, lead, cadmium, and arsenic.

We conducted another pilot clinical trial on a long-term basis. We tracked five subjects who had an elevated total body burden of mercury. We determined this by giving the subjects a DMPS challenge test (a 250-mg IV push) and collected urine over a 6hour period. This is an accurate way of measuring baseline total mercury body burden.

MCP was then orally administered to the five subjects for 4-10 months.

We repeated the DMPS challenge test again-after 4-10 months, depending on the subject. We found that all 5 subjects had dramatic decreases in mercury, with the largest decrease in subjects with the highest total body burden at the beginning of

†Publisher will be Better Health Publishing, Sata Rosa, CA. the study and in those who used MCP for the longest duration. The average decrease for all patients was more than 60 percent. These results are comparable to IV chelation therapies.

We are currently working with researchers in China, due to the high incidence of lead toxicity among children there. In some provinces in China, 65 percent of the children have toxic levels of lead. Even in the "cleaner" Chinese provinces, one is looking at a 15-25 percent toxicity rate-it's a very serious public health problem. High levels of lead in a developing child can lead to neurologic and developmental disorders, bone-marrow separation, cardiomyopathy, anemia, and an increased tendency to cancers.

\section{RM: What are the sources of the lead?}

IE: I think the sources include leaded paints, lead pipes, leaded gasoline, power plants, and industrial waste. Children are exposed to lead in the air they breathe, the water they drink, and-in some cases-lead paint. On the positive side, in initial case reports, $\mathrm{MCP}$ has been shown to substantially reduce blood levels of lead, and we have initiated a study to track 35 Chinese children. I am excited about this because MCP can be potentially change the lives of many children exposed to heavy metals.

\section{RM: What is your specific chelation approach?}

IE: My specific approach is to first chelate in the bloodstream and gut, using MCP, with alginates, on an ongoing basis. After 1-3 months, depending on the patient's heavy metal toxicity levels, I will introduce other chelating agents and nutrients, which will penetrate into the tissue, including the brain.

I combine MCP with modified alginates (sourced from seaweed). They bind to the metals in the gut by surrounding the heavy metals and pulling them through the stool. Low molecular weight alginates can support chelation benefits of MCP because these alginates have a slightly different profile. This combination may enhance systemic chelation but also chelate and avoid reabsorption of heavy metals in the gut. This is especially important with mercury exposure.

The common mistake with respect to chelation is to try to chelate all at once, often with one preparation. It doesn't work as effectively and can cause side-effects. The advantage with MCP and alginates is that they are extremely safe and without the potential side-effects of other chelating agents. As systemic chelating agents, MCP and alginates will decrease the heavy metal concentration in tissues by creating a blood-tissue gradient.

\section{RM: What are your plans for additional work?}

IE: My current focus is on my new book on integrative medicine. Integrative medicine, which has become almost a cliché now, is not simple. It is quite complex. My book is called Beyond Integrative Medicine: The Path of Knowledge and Wisdom. ${ }^{\dagger} \mathrm{I}$ plan to launch the book in the fall of 2005 and explain to a larger audience how integrative medicine can change their lives. 
I will explain how we need to go beyond integrative medicine and use more wisdom and intuition to correctly integrate different healing paths. Another key concept I've learned from my work with cancer patients is how serious illness and disease offers an opportunity for transformation and healing. We have heard of cancer patients who, given a few months to live, have completely turned their lives around and beat cancer while enhancing their quality of life. We can all learn from their experience and use it in our own healing paths!

The heart of the book will incorporate the teachings that I have gained from Tibetan Buddhism and Tibetan medicine, together with my studies of various medical systems. Using the Tibetan Medicine Tree of Knowledge and Wisdom, I will discuss how the roots (the cultivation of nonconceptual wisdom), the trunk (the understanding of health and disease), and the branches and leaves (the knowledge of medical details), come together into fruition. It's this path of knowledge and wisdom that offers a profound experience of healing, health, and personal growth.

RM: Thank you very much for an extremely informative talk today.

IE: You are most welcome.

To order reprints of this article, write to or call: Karen Ballen, ALTERNATIVE \& COMPLEMENTARY THERAPIES, Mary Ann Liebert, Inc., 140 Huguenot Street, 3rd Floor, New Rochelle NY 10801, (914) 740-2100. 